\title{
The guinea pig as an animal model for studying perinatal changes in microvascular function
}

\author{
Rebecca M. Dyson ${ }^{1,2}$, Hannah K. Palliser ${ }^{1,3}$, Meredith A. Kelleher ${ }^{1,3}$, Jonathan J. Hirst ${ }^{1,3}$ and lan M.R. Wright ${ }^{1,2,4}$
}

\begin{abstract}
INTRODUCTION: Microvascular dysfunction, characterized by inappropriate vasodilatation and high blood flow in the peripheral microcirculation, is linked to physiologic instability and poor outcome in neonates. Specifically, preterm neonates have significantly higher levels of baseline microvascular blood flow than term neonates at $24 \mathrm{~h}$ postnatal age. Because of similarities between human and guinea pig endocrine profiles and maturity at birth, we hypothesized that preterm guinea pig neonates would provide a suitable model for studying the mechanisms underlying transitional microvascular function.
\end{abstract}

RESULTS: Guinea pigs that were delivered preterm showed immaturity and had markedly reduced viability. Baseline microvascular blood flow was significantly higher in preterm animals than in term animals. No effect of intrauterine growth restriction or birth weight on baseline microvascular blood flow was observed in either preterm or term animals.

DISCUSSION: These results are consistent with recent clinical findings and support the use of the guinea pig as a suitable model for future studies of the mechanisms underlying perinatal microvascular behavior.

METHODS: Guinea pigs were delivered either prematurely or at term. Laser Doppler flowmetry was used to study microvascular blood flow at $23 \mathrm{~h}$ postnatal age.

ls $\mathrm{n}$ term infants, the circulation undergoes rapid and extensive changes in the initial hours of extrauterine life to allow the infant to effectively deal with extrauterine systemic vascular resistance and hence provide normal and adequate perfusion of tissues (1-3). The transitional circulation of preterm neonates (especially those born at $\leq 30 \mathrm{wk}$ gestation) differs significantly from infants born at full term. In the preterm infant, several crucial physiologic responses to extrauterine life are commonly delayed, allowing for persistence of atrial and ductal shunting and inappropriate blood flow throughout the periphery during the perinatal period (4-8).

Previous studies in preterm infants suggest that abnormal microvascular tone, characterized by inappropriate vasodilatation of the peripheral microvasculature, may contribute to the development of circulatory compromise $(4,8)$. These studies found that the functional integrity of the microvasculature (including appropriate control of vasodilatation) in preterm neonates is significantly altered as compared with neonates born at later gestational ages (GAs). Very preterm neonates (born at 24-28 wk GA) are known to have significantly higher microvascular blood flow at $24 \mathrm{~h}$ postnatal age than preterm neonates born at 29-34 wk GA and neonates born at term. High baseline microvascular blood flow in premature infants is significantly correlated with clinical illness severity and poor outcome in the immediate postnatal period $(4,9)$. Such dysfunction in the microvasculature is also a well-established observation associated with the onset of other causes of multisystem organ failure in neonates (10).

In addition to disorders relating to short gestation, intrauterine growth restriction (IUGR) and low birth weight are also associated with increased neonatal morbidity and mortality, particularly compromise associated with cardiovascular maladaptation $(11,12)$. Previous studies have demonstrated significant changes in peripheral microcirculation during the transitional period; however, the effect of low birth weight or IUGR on baseline microvascular flow has not yet been shown $(13,14)$.

Differences in microvascular blood flow dependent on GA, postnatal age, and sex have been observed in both physiologic and biochemical studies. Physiologic studies have included the assessment of peripheral microcirculation using laser Doppler flowmetry. Recent studies have shown that such evaluation of the peripheral microcirculation is useful for studying cardiovascular changes within the initial extrauterine period and for assessing changes in the microvascular bed of the infant $(15,16)$. These studies also found that measurement of microvascular blood flow is useful for the detection of cardiovascular compromise because the regulatory mechanisms of the peripheral microcirculation contribute to and are involved in the neuroendocrine response to cardiovascular compromise (12). Despite a number of such physiologic studies, the mechanisms underlying these differences are poorly understood. To better understand these mechanisms, an animal model is required to allow the investigation of systems controlling microvascular blood flow because such studies are not possible in the human neonate. Little evidence of the mechanisms controlling

'Mothers and Babies Research Centre, Hunter Medical Research Institute, Newcastle, Australia; ${ }^{2}$ Discipline of Paediatrics and Child Health, School of Medicine and Public Health, University of Newcastle, Newcastle, Australia; ${ }^{3}$ School of Biomedical Sciences and Pharmacy, University of Newcastle, Newcastle, Australia; ${ }^{4}$ Kaleidoscope Neonatal Intensive Care Unit, John Hunter Children's Hospital, Newcastle, Australia. Correspondence: Ian M.R. Wright (ian.wright@hnehealth.nsw.gov.au) 
microvascular maturation exists. The normal ontogeny is not well described, and the development of a suitable animal model for necessary invasive mechanistic investigations is essential to complement ongoing clinical assessments.

The guinea pig has previously been used as an animal model for studying IUGR and lung injury associated with prematurity (17-20). Unlike other laboratory rodents, the guinea pig has a long gestational period. The placental endocrine control and fetal and neonatal endocrine profiles of the guinea pig are comparable to that of the human, making it a particularly suitable model for studying human endocrine function and behavior during pregnancy and early extrauterine life $(21,22)$. The guinea pig is also more precocious than other species of small laboratory animals, with neonates having a highly developed central nervous system, and is thus again more comparable to the human neonate than rat or mouse neonates in terms of perinatal adaptations, because the majority of brain development takes place in utero.

The aim of this study was to establish an animal model of prematurity suitable for studying the mechanisms underlying control and dysregulation of microvascular function in the initial extrauterine period in preterm neonates. It was hypothesized that microvascular blood flow, as measured by laser Doppler flowmetry, would be dependent on GA and sex, as seen in previous human clinical studies $(4,8)$.

\section{RESULTS}

\section{Physical Characteristics}

Spontaneous vaginal delivery $(n=3)$ occurred at $69 \mathrm{~d}$ (67-70 d) gestation (term cesarean section: $69 \mathrm{~d}(68-69 \mathrm{~d})$ gestation; $n=5$ ). No differences in physical characteristics or blood flow analysis were found between term pups delivered by cesarean section or spontaneous vaginal delivery and therefore term pups have been grouped (data not shown). Preterm delivery at $62 \mathrm{~d}(62-63 \mathrm{~d})$ gestation $(n=19)$ resulted in considerable immaturity and decreased viability. Preterm pups had significantly higher mortality rates than pups delivered at term, $P<0.001$ (Figure 1a). In preterm animals, males were found to have higher mortality rates than females (Figure 1b), but this was not statistically significant, $P>0.05$. Henceforth, data presented are for pups surviving to $24 \mathrm{~h}$ postnatal age only, with each dam represented by no more than two male and two female pups (median pups per litter $=2$ (range 1-4)). Therefore, each group is represented by a minimum of six litters. Physical characteristics of surviving pups are presented in Table 1. Surviving preterm pups required respiratory support, including surfactant administration and continuous positive air pressure with positive pressure ventilation, incubation, and tube feeding. As a reflection of this, the neonatal monitoring scores of preterm pups over the 24-h study period were significantly lower (9.2 (8.0-9.9)) than those of term animals (12 (11.9-12)), $P<0.001$. Birth weight was significantly reduced in preterm pups $(66.4 \mathrm{~g}(53.9-70.4 \mathrm{~g}))$ as compared with term animals (76.3 g (68.0-92.1 g) ), $P<0.001$. No significant sex difference was found in body weight between males and females in either preterm or term animals.

\section{Intrauterine Growth Restriction}

Pups were classified as growth restricted based on calculation of brain-to-liver weight ratio. IUGR resulted in significantly reduced birth weights of both sexes of neonates in both the term $(P=0.001)$ and preterm $(P<0.001)$ groups,
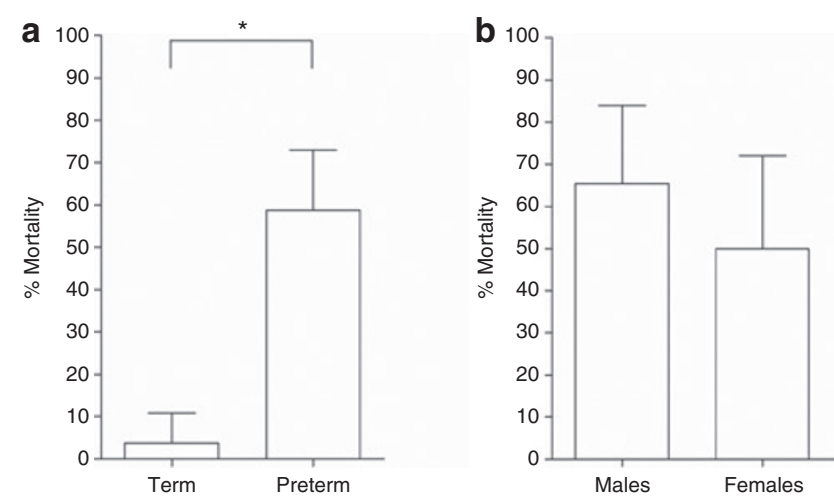

Figure 1. Mortality rates at $24 \mathrm{~h}$ postnatal age in term and preterm animals. (a) As compared with animals delivered at full term, preterm delivery resulted in significantly decreased viability despite ongoing respiratory, thermal, and nutritional support. ${ }^{*}$ Denotes significant difference $(P<0.001)$. (b) Mortality was greater in preterm males than females. Although not statistically significant, the increased male mortality is comparable to that seen in large cohorts of human preterm neonates. Data are expressed as $\%$ mortality $\pm 95 \%$ confidence intervals.

Table 1. Physical characteristics of neonates

\begin{tabular}{|c|c|c|c|c|}
\hline & \multicolumn{2}{|c|}{ Preterm group } & \multicolumn{2}{|c|}{ Term group } \\
\hline & Male $(n=15)$ & Female $(n=16)$ & Male $(n=13)$ & Female $(n=11)$ \\
\hline $\mathrm{GA}(\mathrm{d})$ & $62.0(62.0-63.0)$ & $62.0(62.0-63.2)$ & 69 & 69 \\
\hline Cesarean section delivery $(n, \%)$ & $15(100 \%)$ & $16(100 \%)$ & $9(69.2 \%)$ & $7(63.6 \%)$ \\
\hline IUGR $(n, \%)$ & $5(33.3 \%)$ & $5(31.3 \%)$ & $5(38.5 \%)$ & $4(36.4 \%)$ \\
\hline Animal temperature $\left({ }^{\circ} \mathrm{C}\right)$ & $36.9(36.5-37.7)$ & $36.8(35.5-37.7)$ & $37.1(35.7-37.4)$ & $36.8(36.4-37.0)$ \\
\hline
\end{tabular}

GA, gestational age; IUGR, intrauterine growth restriction.

Data presented as median (interquartile range) or number (\%) for pups surviving to laser Doppler analysis of blood flow ( $23 \mathrm{~h}$ postnatal age). Neonatal monitoring score presented is average score over $24 \mathrm{~h}$; animal temperatures presented are those recorded at time of laser Doppler. 


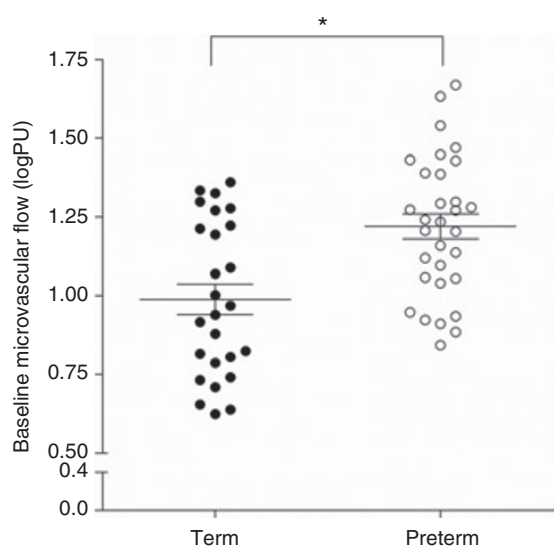

Figure 2. Baseline microvascular blood flow in guinea pig neonates. Baseline microvascular blood flow, expressed as the log of arbitrary perfusion units (logPU), was measured using laser Doppler flowmetry in term (filled circles, $n=26$ ) and preterm (open circles, $n=31$ ) male and female animals at $23 \mathrm{~h}$ postnatal age. Baseline microvascular blood flow was significantly higher in preterm animals than in term controls. Data are expressed as mean \pm SEM. ${ }^{*}$ Denotes significant difference $(P<0.001)$.

and asymmetrical growth restriction was also evidenced by significantly increased brain weight to body weight ratios in IUGR as compared with non-IUGR pups in both the term $(P<0.001)$ and preterm $(P<0.001)$ groups (data not shown). Incidence of IUGR was not significantly different between the term and preterm groups ( $32.3 \%$ and $37.5 \%$, respectively, $P=0.78)$, and rates did not differ between sexes in either GA group (Table 1).

\section{Laser Doppler Flowmetry}

Baseline microvascular flow was significantly higher in preterm animals $(1.22 \pm 0.04 \log \mathrm{PU})$ than in term animals $(0.99 \pm 0.05$ $\log \mathrm{PU} ; P<0.001$ ) at $23 \mathrm{~h}$ postnatal age (Figure 2). Baseline flow did not differ significantly between sexes in either gestational group (data not shown). Baseline microvascular blood flow was not significantly different between term IUGR $(1.001 \pm 0.08$ $\log \mathrm{PU})$ and non-IUGR $(1.005 \pm 0.07 \log \mathrm{PU} ; P=0.97)$ or preterm IUGR $(1.223 \pm 0.07 \log P U)$ and non-IUGR $(1.218 \pm 0.05$ $\log P U)$ neonates $(P=0.95)$. No significant relationship between birth weight and baseline microvascular flow was found to exist for either term $(r=0.165)$ or preterm $(r=0.148)$ animals.

\section{DISCUSSION}

The key finding of this study is that preterm guinea pig neonates have significantly higher levels of baseline microvascular flow than those delivered at term; however, IUGR status and birth weight show no relationship with microvascular blood flow at $23 \mathrm{~h}$ postnatal age. This finding is consistent with observations made in human neonates born at earlier GAs (24-28 wk gestation) as compared with neonates born at later GAs (29-34 wk gestation) $(4,9)$.

One of the major difficulties in establishing an animal model to represent the preterm human neonate is determining developmental age equivalency of the species and humans. On the basis of physical characteristics and mortality in our preterm animals, we suggest that the neonatal guinea pig delivered at $62 \mathrm{~d}$ GA is an appropriate model for studying microvascular adaptations in preterm humans during the immediate postnatal period. Normal gestation in our guinea pig population is approximately $71 \mathrm{~d}(22)$. Preterm neonates were delivered at $62 \mathrm{~d} \mathrm{GA}$, the approximate equivalent of a human infant born at $34.5 \mathrm{wk}$ completed gestation. However, the demonstrated decreased viability and significant physiologic immaturity of these preterm neonates suggest an earlier age equivalency. This is supported by previous studies in the guinea pig that have assessed lung development in the fetal guinea pig and suggest that pups born before $66 \mathrm{~d}$ GA show considerable lack of development, demonstrated by lung growth and development, and that guinea pig neonates born at $<88 \%$ completed gestation cannot survive with non-invasive interventions (23). These findings are consistent with the significantly increased mortality of preterm guinea pig neonates observed in this study. Previous studies have concluded that lung growth and development parallels the overall development of a species at birth (24). In a previous study of lung development, fetal guinea pigs at $61 \mathrm{~d}$ gestation had lung morphology indicative of the saccular stage of lung development, which is seen in the human neonate from approximately $24 \mathrm{wk}$ of GA through to term (23). Viability and support levels in our neonatal guinea pigs suggest that they are more physiologically equivalent to a human infant born at approximately $29 \mathrm{wk}$ completed gestation because approximately half of the infants born at this GA could be expected to survive with only our limited interventions.

It has long been established that male infants born prematurely are at an increased risk of poor outcome as compared with females of the same GA $(8,25-27)$. This increased death rate seems to be associated with cardiorespiratory compromise (28). The mechanisms underlying this increased risk are not yet understood, although sex differences in physiologic responses to the fetal-to-neonatal transition are well described (29-32). Our mortality rates for males and females in the preterm cohort are in line with such clinical observations. Although the difference in mortality between male and female neonates was not statistically significant (Figure 1b), it suggests that the preterm guinea pig delivered at this GA may be comparable to the human preterm neonate in terms of sexual dimorphisms seen in viability at earlier GAs. Significant loss of viability in male as compared with female preterm neonates has been reported in large cohort human studies (33), and power analyses of our data suggest that a much larger number of litters $(n=100)$ would have to be included in our animal cohort for this result to reach significance at the same effect size as in the human population. This is neither practical nor ethical for animal studies of this nature.

In humans, preterm male infants have been shown to have significantly higher baseline levels of microvascular flow than females. This sexual dimorphism is evident at $24 \mathrm{~h}$ postnatal age, but it is no longer observed when the infant reaches $72 \mathrm{~h}$ postnatal age (8). Although we did not observe a significant sex difference or an effect of IUGR on baseline microvascular blood flow, we propose that this may be a 
survival effect or a postnatal age effect. Further studies will need to investigate these factors to determine the usefulness of this model for studying sexual dimorphism in the control of extrauterine microvascular blood flow. Such studies will need to investigate microvascular function at earlier time points to assess whether neonates with higher microvascular flow, which is known to correlate with adverse outcome, are not represented by the group of neonates surviving to $24 \mathrm{~h}$. Earlier time points also need to be considered because the transition from fetal to neonatal circulatory systems is a rapid and extensive process, and it may be that $24 \mathrm{~h}$ postnatal age in the neonatal guinea pig is outside the window of adaptation when maximal dysregulation resulting in circulatory compromise and adverse outcome could be expected to occur.

Our results are consistent with those of previous clinical studies, which show that preterm birth, but not IUGR or low birth weight, is associated with high baseline microvascular blood flow at $24 \mathrm{~h}$ postnatal age. Further studies at earlier postnatal ages have great potential to further our understanding of the relationship between dysregulation of the peripheral microvasculature and illness severity or mortality. Taken together, results from this study support the use of the preterm guinea pig as an animal model for studying perinatal microvascular changes in the initial extrauterine period. This will allow mechanistic studies to better understand the control of microvascular flow in the preterm neonate during this period of circulatory compromise.

\section{Ethics}

All procedures were approved by the University of Newcastle Animal Care and Ethics Committee and were performed in accordance with the Australian Code of Practice for the Care and Use of Animals for Scientific Purposes.

\section{METHODS}

\section{Animals}

The Research Support Unit of the University of Newcastle supplied time-mated, pregnant outbred, tricolor guinea pigs. Male and female offspring were used for this study, with no dam represented by more than two pups of each sex in any group.

\section{Spontaneous Term Delivery}

For spontaneous vaginal delivery to occur, the pubic symphysis of the pregnant dam must relax and open, allowing for passage of the fetus. Detectable separation of this joint for 2 consecutive days indicates imminent onset of labor. Dams were monitored for delivery daily throughout late gestation and then hourly using an infrared camera after pubic symphysis separation. Pups were separated from the dam immediately after delivery.

\section{Cesarean Section Delivery}

Pregnant dams were allocated to either preterm or term delivery. For preterm delivery, a cesarean section delivery was performed between 62 and $64 \mathrm{~d} \mathrm{GA}$ (full term: approximately $71 \mathrm{~d}(22)$ ). As a percentage of completed gestation (87\%), this is the equivalent of a human infant born in the late preterm period (approximately $34.5 \mathrm{wk} \mathrm{GA}$ ). This time point was chosen because it is the earliest GA that guinea pig pups can be kept alive with non-invasive support (23). Guinea pigs have been shown to be markedly resistant to glucocorticoids such as betamethasone, with significantly decreased glucocorticoid receptor binding affinity (34). For this reason, $1 \mathrm{mg} / \mathrm{kg}$ betamethasone (as compared with an average dose of $0.2 \mathrm{mg} / \mathrm{kg}$ used clinically in human pregnancies) was administered to pregnant dams subcutaneously at 48 and $24 \mathrm{~h}$ before delivery to stimulate fetal lung maturation. This dosage has been shown to exert effects on the guinea pig similar to those seen clinically in humans $(35,36)$. Term cesarean sections were performed at $69 \mathrm{~d}$ gestation or earlier if the pubic symphysis had been open for 2 consecutive days. The dam was anesthetized for surgical delivery by administering $4 \%$ isoflurane in medical grade oxygen using chamber and mask inhalation for $15 \mathrm{~min}$ before delivery. At delivery, the uterus was exposed by means of an incision made down the midline of the ventral abdomen and removed from the abdominal cavity maintaining blood supply. Pups were rapidly removed from the uterus and transferred to a warm bed for immediate resuscitation.

\section{Neonatal Respiratory Support}

Lung fluid was cleared by suction from the airways of pups. Preterm pups were then placed in a supine position and administered $50 \mu \mathrm{l}$ surfactant $(80 \mathrm{mg} / \mathrm{ml}$; Poractant Alfa, Ascent, South Melbourne, Australia) into the oropharynx with a gasp induced by nasal occlusion. Pups then received continuous positive air pressure with positive pressure ventilation as required, administered in a $1: 1$ ratio of oxygen and medical air at a rate of $81 / \mathrm{min}$. A Neopuff Infant T-Piece Resuscitator (Fisher \& Paykel Healthcare, Melbourne, Australia) with a positive end-expiratory pressure of $7 \mathrm{~cm} \mathrm{H}_{2} \mathrm{O}$ was used to establish and maintain functional residual capacity, and when required, mechanical ventilation with a peak inflation pressure of $20 \mathrm{~cm} \mathrm{H}_{2} \mathrm{O}$ was administered. In pups with no spontaneous breathing present after birth, ventilation was started with an initial sustained inflation with a peak inflation pressure of $20 \mathrm{~cm} \mathrm{H}_{2} \mathrm{O}$ for $20 \mathrm{~s}(37)$.

\section{Neonatal Monitoring}

Once the pups were stable, birth weight was recorded and they were maintained in a humidified incubator at temperatures of $34-38^{\circ} \mathrm{C}$ as required for maintenance of neonatal body temperature for the duration of the study period. Rectal temperature was measured every $2 \mathrm{~h}$. Because pups were delivered by non-recovery surgery of pregnant dams, frequent feeding and monitoring of the neonates was required. Pups were monitored every $2 \mathrm{~h}$, and clinical states were recorded using a novel neonatal monitoring score based on three criteriarespiration, posture, and alertness/movement. A maximum score of 4 was assigned for each criterion, thus a maximum total of 12 . Scores of 3 and below were categorized as very poor, 4-6 as poor, 7-9 as good, and $10-12$ as very good. Preterm pups were also fed every $2 \mathrm{~h}$ with commercial guinea pig milk formula using a single lumen polyvinylchloride orogastric tube. For term pups, the milk formula was titrated intraorally.

\section{Laser Doppler Flowmetry}

Laser Doppler readings were recorded at $23 \mathrm{~h}$ postnatal age, no less than $30 \mathrm{~min}$ after the last feeding of the pups. The PeriFlux System 5001 (Perimed, Jarfalla, Sweden) with a small straight probe (Probe 407) and miniature probe holder (PH07-4) was used. The probe was applied gently to the rear of the pad of the hind foot to establish baseline microvascular flow. A baseline microvascular flow was considered established when backscatter was stable and heart rate and rhythmical flow could be visualized within the PeriSoft (PSW2.0, Perimed AB) display for at least $30 \mathrm{~s}$. The microvasculature was then subjected to occlusion for $1 \mathrm{~min}$ (achieved by applying pressure to occlude blood flow). Biological baseline (reported value) was determined by subtracting the minimum value during occlusion ("biozero") from the mean value for the baseline reading. Microvascular blood flow data were recorded and analyzed using custom PeriSoft software and are expressed in arbitrary perfusion units (PU) (38). Only recording sequences free from movement artifacts were analyzed. All laser Doppler studies were performed by a single investigator (R.D.). 


\section{Postmortem Tissue Collection}

Body, brain, and liver weights were collected postmortem at $24 \mathrm{~h}$ postnatal age. IUGR was defined by a brain-to-liver weight ratio $>0.9$. This has previously been defined as a marker of asymmetric growth restriction in the guinea pig (18). To compare with human data when liver weights are not known and small-for-GA infants with head sparing are diagnosed as having IUGR through head circumference:body weight ratio, the brain:body weight ratio was also calculated for neonates.

\section{Statistics}

Prism 4 for MacOSX (GraphPad Software, La Jolla, CA) was used for statistical analyses and generating graphs. Unless otherwise stated, data for physical characteristics are presented as median (interquartile range) and analyzed using two-way ANOVA. Incidence and mortality data were analyzed using Fisher's exact test. Laser Doppler flowmetry data were not normally distributed and were therefore log-transformed for further analysis (logPU). Differences between groups for microvascular blood flow logged data are presented as mean \pm SEM and were analyzed by unpaired $t$ test or two-way ANOVA as indicated. Significance level was set at $P<0.05$ for all data examined.

\section{STATEMENT OF FINANCIAL SUPPORT}

This study was funded by a John Hunter Hospital Charitable Trust Project Grant awarded to I.M.R.W. and R.M.D. R.M.D. was supported by a Hunter Children's Research Foundation Scholarship.

\section{REFERENCES}

1. Bhutani VK. Extrauterine adaptations in the newborn. Semin Neonatol 1997;2:1-12.

2. Sinha SK, Donn SM. Fetal-to-neonatal maladaptation. Semin Fetal Neonatal Med 2006;11:166-73.

3. Rudolph AM. Circulatory adjustments after birth: effects on ventricular septal defect. Br Heart J 1971;33:Suppl:32-4.

4. Stark MJ, Clifton VL, Wright IM. Microvascular flow, clinical illness severity and cardiovascular function in the preterm infant. Arch Dis Child Fetal Neonatal Ed 2008;93:F271-4.

5. Evans N. Assessment and support of the preterm circulation. Early Hum Dev 2006;82:803-10.

6. Evans NJ, Archer LN. Postnatal circulatory adaptation in healthy term and preterm neonates. Arch Dis Child 1990;65(1 Spec No):24-6.

7. Kluckow M. Low systemic blood flow and pathophysiology of the preterm transitional circulation. Early Hum Dev 2005;81:429-37.

8. Stark MJ, Clifton VL, Wright IM. Sex-specific differences in peripheral microvascular blood flow in preterm infants. Pediatr Res 2008;63:415-9.

9. Stark MJ, Clifton VL, Wright IM. Neonates born to mothers with preeclampsia exhibit sex-specific alterations in microvascular function. Pediatr Res 2009;65:292-5.

10. Smith SD, Tagge EP, Hannakan C, Rowe MI. Characterization of neonatal multisystem organ failure in the surgical newborn. J Pediatr Surg 1991;26:494-7; discussion 497-9.

11. Gilbert WM, Danielsen B. Pregnancy outcomes associated with intrauterine growth restriction. Am J Obstet Gynecol 2003;188:1596-9; discussion 1599-601.

12. Ishiguro A, Sekine T, Kakiuchi S, et al. Skin and subcutaneous blood flows of very low birth weight infants during the first 3 postnatal days. J Matern Fetal Neonatal Med 2010;23:522-8.

13. Martin $\mathrm{H}$, Lindblad B, Norman $M$. Endothelial function in newborn infants is related to folate levels and birth weight. Pediatrics 2007;119:1152-8.

14. Norman M, Martin H. Preterm birth attenuates association between low birth weight and endothelial dysfunction. Circulation 2003;108: 996-1001.

15. Hanssler L, Roll C, Breukmann H. [Laser Doppler flowmetry in newborn infants with low birth weight. The effect of differences in humidity on peripheral circulation]. Klin Padiatr 1992;204:359-61.
16. Martin H, Norman M. Skin microcirculation before and after local warming in infants delivered vaginally or by caesarean section. Acta Paediatr 1997;86:261-7.

17. Lafeber HN, Rolph TP, Jones CT. Studies on the growth of the fetal guinea pig. The effects of ligation of the uterine artery on organ growth and development. J Dev Physiol 1984;6:441-59.

18. Carter AM, Kingston MJ, Han KK, Mazzuca DM, Nygard K, Han VK. Altered expression of IGFs and IGF-binding proteins during intrauterine growth restriction in guinea pigs. J Endocrinol 2005;184:179-89.

19. Langley SC, Kelly FJ. Depletion of pulmonary glutathione using diethylmaleic acid accelerates the development of oxygen-induced lung injury in term and preterm guinea-pig neonates. J Pharm Pharmacol 1994;46: 98-102.

20. McKendry AA, Palliser HK, Yates DM, Walker DW, Hirst JJ. The effect of betamethasone treatment on neuroactive steroid synthesis in a foetal Guinea pig model of growth restriction. J Neuroendocrinol 2010;22: $166-74$.

21. Kaufmann P, Davidoff M. The guinea-pig placenta. Adv Anat Embryol Cell Biol 1977;53:5-91.

22. Palliser HK, Zakar T, Symonds IM, Hirst JJ. Progesterone receptor isoform expression in the guinea pig myometrium from normal and growth restricted pregnancies. Reprod Sci 2010;17:776-82.

23. Sosenko IR, Frank L. Lung development in the fetal guinea pig: surfactant, morphology, and premature viability. Pediatr Res 1987;21:427-31.

24. ENGEL $S$. The structure of the respiratory tissue in the newly-born. Acta Anat (Basel) 1953;19:353-65.

25. Naeye RL, Burt LS, Wright DL, Blanc WA, Tatter D. Neonatal mortality, the male disadvantage. Pediatrics 1971;48:902-6.

26. Stephens BE, Vohr BR. Neurodevelopmental outcome of the premature infant. Pediatr Clin North Am 2009;56:631-46.

27. Fanaroff AA, Stoll BJ, Wright LL, et al.; NICHD Neonatal Research Network. Trends in neonatal morbidity and mortality for very low birthweight infants. Am J Obstet Gynecol 2007;196:147 e1-8.

28. Henderson-Smart DJ, Hutchinson JL, Donoghue DA, Evans NJ, Simpson JM, Wright I; Australian and New Zealand Neonatal Network. Prenatal predictors of chronic lung disease in very preterm infants. Arch Dis Child Fetal Neonatal Ed 2006;91:F40-5.

29. Baenziger O, Jaggi JL, Mueller AC, et al. Cerebral blood flow in preterm infants affected by sex, mechanical ventilation, and intrauterine growth. Pediatr Neurol 1994;11:319-24.

30. Chen SJ, Vohr BR, Oh W. Effects of birth order, gender, and intrauterine growth retardation on the outcome of very low birth weight in twins. J Pediatr 1993;123:132-6.

31. Davis M, Emory E. Sex differences in neonatal stress reactivity. Child Dev 1995;66:14-27.

32. Hoffman EL, Bennett FC. Birth weight less than 800 grams: changing outcomes and influences of gender and gestation number. Pediatrics 1990;86:27-34.

33. Ingemarsson I. Gender aspects of preterm birth. BJOG 2003;110:Suppl 20:34-8

34. Fuller PJ, Smith BJ, Rogerson FM. Cortisol resistance in the New World revisited. Trends Endocrinol Metab 2004;15:296-9.

35. Owen D, Matthews SG. Repeated maternal glucocorticoid treatment affects activity and hippocampal NMDA receptor expression in juvenile guinea pigs. J Physiol (Lond) 2007;578(Pt 1):249-57.

36. Li T, Koshy S, Folkesson HG. IL-1beta-induced cortisol stimulates lung fluid absorption in fetal guinea pigs via SGK-mediated Nedd4-2 inhibition. Am J Physiol Lung Cell Mol Physiol 2009;296:L527-33.

37. te Pas AB, Siew M, Wallace MJ, et al. Effect of sustained inflation length on establishing functional residual capacity at birth in ventilated premature rabbits. Pediatr Res 2009;66:295-300.

38. Leahy MJ, de Mul FF, Nilsson GE, Maniewski R. Principles and practice of the laser-Doppler perfusion technique. Technol Health Care 1999; $7: 143-62$. 